\title{
De novo peroxisome biogenesis revisited
}

\author{
Marten Veenhuis and Ida J. van der Klei* \\ Molecular Cell Biology, Groningen Biomolecular Sciences and Biotechnology Institute, University of Groningen, The Netherlands. \\ * Corresponding Author: Ida J. van der Klei, P.O. Box 11103; 9700 CC Groningen, The Netherlands; Tel: +31 50 363 2179/2400; Fax: \\ +31 50363 2348; E-mail: i.j.van.der.klei@rug.nl
}

\begin{abstract}
We describe an alternative peroxisome formation pathway in yeast pex3 and pex19 cells, which relies on the existence of small peroxisomal remnants that are present in these cells. This groundbreaking result challenges current models prescribing that peroxisomes derive de novo from the ER. Our data also has major implications for the sorting pathway of specific peroxisomal membrane proteins (PMPs). We propose a novel sorting pathway for the PMPs Pex13 and Pex14 that is independent of the known Pex3/Pex19 machinery.
\end{abstract}

Peroxisomes are crucial, multifunctional organelles the abundance and function of which continuously adapt to satisfy cellular needs. The development of these organelles is strongly debated. Current models differ from multiplication by fission via dynamin-related protein (Drp) dependent fission machineries, which are well documented now. An alternative model prescribes that most, if not all, organelles form de novo from the endoplasmic reticulum (ER). De novo peroxisome biogenesis is most studied following functional complementation of PEX3 deletion (pex3) stains, which so far were assumed to fully lack peroxisomal membrane structures. Fluorescence microscopy (FM) analysis of complemented cells revealed that newly synthesized Pex3GFP sorts to the ER, concentrates in foci followed by the formation of a pre-peroxisomal structure, which pinches off and develops into a nascent peroxisome. Alternatively, two (in Saccharomyces cerevisiae) or multiple (in Yarrowia lipolytica) types of vesicles have been proposed developing from the ER which subsequently fuse to form a nascent peroxisome.

We have now shown that these models are no longer generally valid as Hansenula polymorpha and $S$. cerevisiae pex3 cells, other than generally anticipated, do contain small peroxisomal membrane remnants (ghosts), which are the target for reintroduced Pex3 and the template for subsequent peroxisome formation. Similar observations were made in pex19 cells of both species although the pex19 vesicles differed from those present in pex3 cells in that they contained, besides Pex13, Pex14 and Pex8, also Pex3. Pex13 and Pex14 are key components of the matrix protein receptor docking complex. In the membrane remnants small amounts of matrix protein were present suggesting that Pex13 and Pex14 were correctly inserted and functional as receptor docking site. The low matrix content may be explained in that the proteins of the receptor recycling system (including the RING finger proteins) were not present on these structures, thereby preventing recycling of the PTS1 receptor Pex5. Indeed, Pex5 was found associated with the vesicles, whereas other PMPs, e.g. PMP47 and the RING finger proteins, were unstable and present in low amounts in the cytosol.

These findings have major implications for the current concepts of peroxisome de novo formation. These models invariably prescribe that the ER is the membrane template for de novo synthesis of which we showed that this is no longer generally valid. Most likely, this can be explained by the relatively low resolution of the fluorescence microscopy (FM) techniques used. Also, in most cases Pex3 synthesis was driven by the strong, inducible galactose promoter giving rise to relative high Pex3 protein levels at the initial stages of peroxisome re-induction compared to the low levels produced under control of the endogenous PEX3 promoter. This initial overexpression effect most likely resulted in mislocalization of excess Pex3-GFP at the ER. As the vesicular structures in pex3 and pex19 cells are generally localized in close vicinity of the ER, the resolution of FM is insufficient for discriminating the vesicular structures from the ER. The latter could only be achieved with the high resolution microscopy techniques we applied. Possibly, the Pex3 puncta that have been described before to be localized at the ER at the initial stage of pre-peroxisome formation in fact represent the vesicular structures. Using Pex3 synthesis driven under the control of the endogenous PEX3 promoter we have never seen localization of Pex3 to

MICROREVIEW on: Knoops K, Manivannan S, Cepinska MN, Krikken AM, Kram AM, Veenhuis M, van der Klei IJ (2014). Preperoxisomal vesicles can form in the absence of Pex3. J Cell Biol 204(5):659-68. doi: 10.1083/jcb.201310148.

doi: $10.15698 /$ mic2014.04.138

Received: 21.03.2014; Accepted 26.03.2014, Published 01.04.2014.

Keywords: yeast, peroxisomes, de novo peroxisome formation, peroxisome deficient mutant, endoplasmic reticulum. 
A

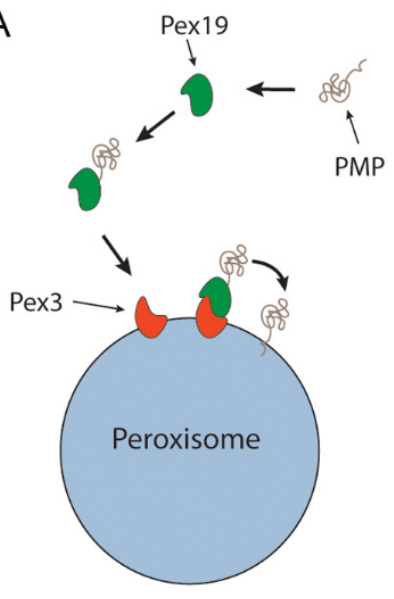

B

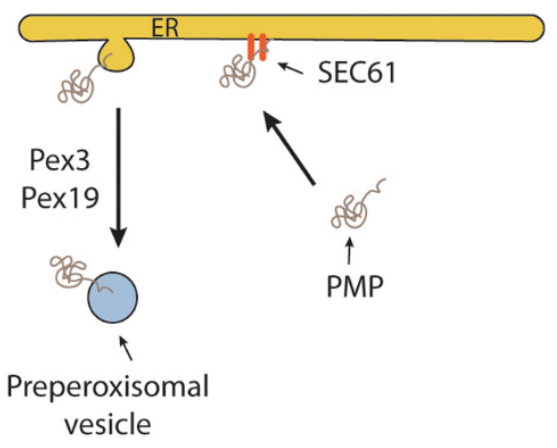

FIGURE 1: Current PMP sorting models. (A) PMP sorting requires the Pex3/Pex9 sorting machinery. In this classical model Pex19 functions as receptor/chaperone to sort newly synthesized PMP molecules to the peroxisomal docking site Pex3 followed by PMP insertion in the membrane by yet unknown mechanisms. (B) PMP sorting requires the ER. According to this model peroxisomes are formed from the ER. PMPs are first inserted in the ER dependent of the Sec61 machinery and subsequently incorporated in single or multiple vesicles that are separated from the ER via the function of Pex3 and Pex19. In case of multiple vesicles, these fuse to form a pre-peroxisomal vesicle. the ER but did so when synthesis was under control of the strong alcohol oxidase promoter.

Together, our observations convincingly show that the $E R$ is not the initial membrane template for peroxisome formation in pex3 and pex19 mutants and hence that upon complementation of these mutants with the corresponding genes the organelles are formed from a pre-peroxisomal structure and not de novo (i.e. from a template unrelated to peroxisomes). It also uncovered a novel mechanism of PMP sorting. Towards this, so far two main models existed, explaining the absence of peroxisomal membrane structures in pex3 and pex19 cell (Fig. 1). One of these proposes that, upon synthesis in the cytosol on free ribosomes, PMPs are sorted to the target organelle via the Pex19/Pex3 sorting machinery (Fig. 1A). In this machinery Pex19 func- tions as receptor/chaperone to bind newly synthesized PMPs and transports them to Pex3, serving as membrane docking site. The second model (Fig. 1B) prescribes that all PMPs travel via the ER, from which pre-peroxisomal vesicles are formed. In this model Pex3 and Pex19 are required for exit of the PMPs from the ER in vesicles. Together, our novel data provide new conceptual insight into peroxisome biogenesis. First, it changes the concept of de novo peroxisome formation from the ER, as this machinery most likely does not exist. This does not imply that the ER may not play a role at all, but most likely solely serves a function in membrane lipid supply. Also a role in the formation in the vesicular structures present in pex 3 and pex 19 cells cannot be excluded (Fig. 2), but our data clearly do not fit with a model that all peroxisomes arise from the ER. Possibly,

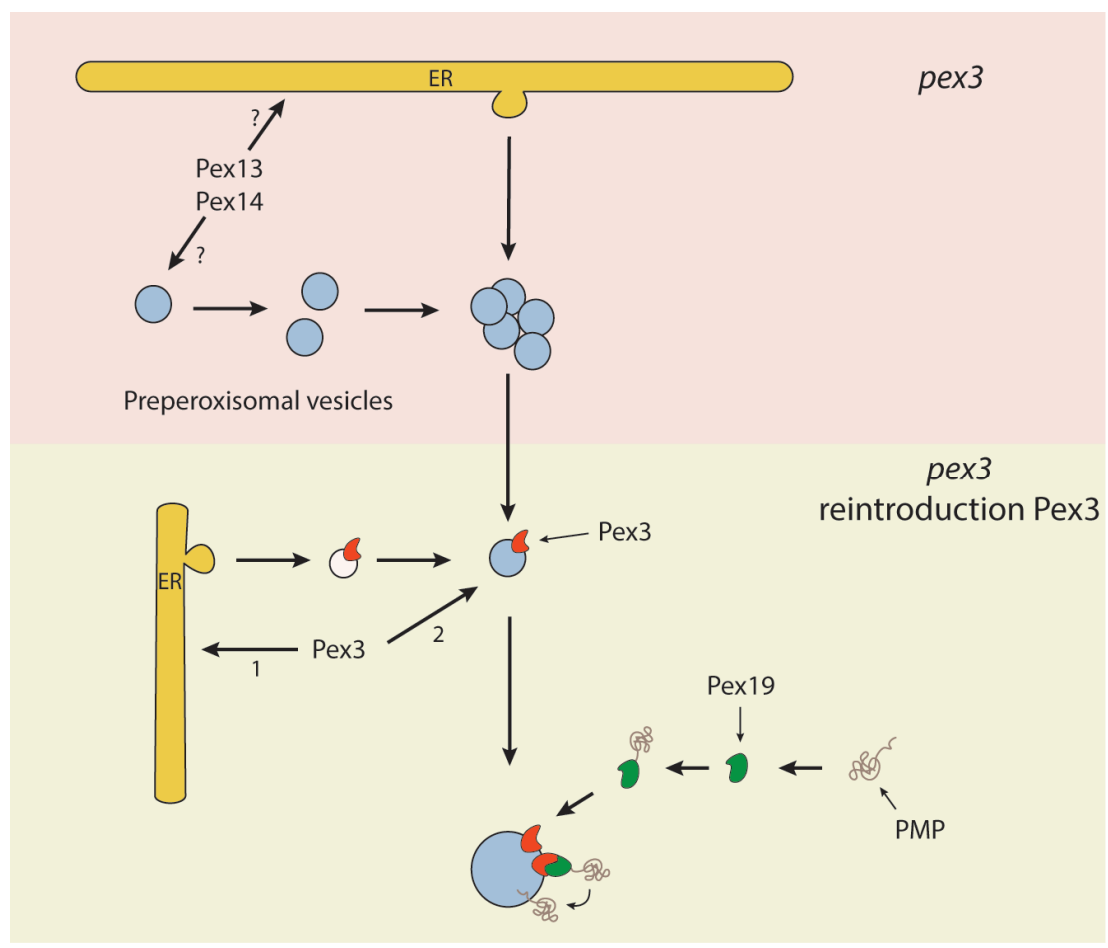

FIGURE 2: Schematic overview of the novel peroxisome biogenesis pathway. The upper pink part represents the presence of preperoxisomal vesicles, containing Pex13 and Pex14, in yeast PEX3 deletion cells. These vesicles may be autonomous and proliferate by fission or form from the $E R$ in a Pex3/Pex19 independent manner. Upon reintroduction of Pex3 (lower part in green), the protein most likely directly travels to the pre-peroxisomal vesicles or alternatively, reaches these via ER derived vesicles. After incorporation of Pex3 in the pre-peroxisomal vesicles, the other PMPs insert into the vesicles via the Pex3/Pex19 dependent docking machinery (see also Fig. 1A). 
peroxisome inheritance mutants are favorite models to study de novo peroxisome biogenesis provided that these cells do not contain the vesicular structures observed in pex3 and pex19 mutants. Our data confirm that most PMPs, which are unstable and soluble in the mutant cells, rapidly stabilize and sort to the vesicular structures in a Pex19/Pex3 dependent manner upon functional complementation of the corresponding pex3 or pex19 mutant (Fig. 2). Hence, these are not sorted via the ER. Furthermore, in pex3 and pex19 cells the vesicular membrane structures contained Pex13, Pex14 and Pex8 indicating that these proteins reach these structures independent of Pex3 and Pex19. How these proteins reach their target membrane is yet fully unclear but possibly may involve the function of the ER (Fig.2). Also, we have never seen Pex3 accumulating in the ER when produced from its own promoter. However, the residence time of this protein in the ER may be too short to track its routing via FM. Therefore, routing of Pex3 requires further investigation using advanced microscopy and biochemical techniques.

The present work has opened new avenues to unravel the principles of peroxisome biogenesis. Many questions remain. An urgent question to solve is on the origin of the vesicular structures in pex3 and pex19 cells. Are they au- tonomous or do they derive from the ER? What are the protein components essential for their formation? Yet, the first component has been identified in two independent studies. We and the Hartig group identified Pex25 as being essential for de novo peroxisome formation in yeast pex 3 cells and in young buds of inp2 cells that lacked peroxisomes through an inheritance defect.

\section{ACKNOWLEDGMENTS}

We thank Rinse de Boer for preparing the figures.

\section{CONFLICT OF INTEREST}

The authors declare no competing interests.

\section{COPYRIGHT}

(C) 2014 Veenhuis and van der Klei. This is an open-access article released under the terms of the Creative Commons Attribution (CC BY) license, which allows the unrestricted use, distribution, and reproduction in any medium, provided the original author and source are acknowledged.

Please cite this article as: Marten Veenhuis and Ida J. van der Klei (2014). De novo peroxisome biogenesis revisited. Microbial Cell 1(4): 128-130. doi: 10.15698/mic2014.04.138 